\title{
EDITORIAL
}

\section{Is COPD in adulthood really so far removed from early development?}

\author{
W. Shi and D. Warburton
}

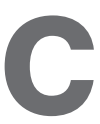
hronic obstructive pulmonary disease (COPD), including chronic bronchitis and emphysema, is commonly thought of as an adult onset lung disease most often seen in aging people with a tobacco smoking history [1]. Yet, conversely, only $\sim 20 \%$ of cigarette smokers develop full-blown emphysema, while COPD may also be seen in nonsmokers. Therefore, genetic susceptibility is a hot topic in recent COPD research. A wealth of information has been accumulating regarding genetic polymorphisms and the incidence and severity of COPD in patients. However, the mechanisms by which these genetic alterations contribute to COPD pathogenesis are as yet barely understood. Accumulated research evidence from gene knockout and transgenic mouse models suggest that some of these genetic mutations may affect lung developmental processes in early life, which may later function as an antecedent of apparently adult onset COPD. For example, conventional gene knockout of latent transforming growth factor- $\beta$ binding protein 4 or Smad3 results in retarded lung alveogenesis during early postnatal life with further subsequent alveolar enlargement [2,3]. Mutations of these genes interfere with transforming growth factor (TGF)- $\beta$ signalling, which regulates lung growth in early life. A lung with developmental abnormalities may thus be predisposed to further disruption of lung homeostasis that may be caused by imbalanced protease/anti-protease activities, for example matrix metallopeptidase 9. However, blockade of TGF- $\beta$ signalling in lung epithelial cells alone is not sufficient for spontaneous development of emphysematous pathology [4], suggesting that disruption of TGF- $\beta$ signalling in multiple systems including immune regulation, contributes to the subsequent development of alveolar destruction. As a further multisystem protease/antiprotease balance example, a disintegrin and protease gene, disintegrin and metalloproteinase domain-containing protein 33 , has been identified as relevant in lung development [5], in lung function in the pre-school years [6], as well as in asthma and COPD [7, 8]. Interestingly, the studies of DiDON et al. [9], as published in this issue, report

Developmental Biology and Regenerative Medicine Program, The Saban Research Institute of Childrens Hospital Los Angeles, Keck School of Medicine and School of Dentistry, University of Southern California, Los Angeles, CA, USA.

CORRESPONDENCE: D. Warburton, Developmental Biology and Regenerative Medicine Program, The Saban Research Institute of Childrens Hospital Los Angeles, Keck School of Medicine and School of Dentistry, University of Southern California, Los Angeles, CA, USA. E-mail: dwarburton@chla.usc.edu that lung epithelium-specific deletion of the transcription factor CCAAT-enhancer binding protein $\alpha$ disrupts normal fetal mouse lung development, including altered airway and lung cell differentiation as well as vascularity in early life. This is followed by spontaneous development of chronic bronchial inflammation, together with excess mucus production, as well as emphysema in adulthood, a phenotype that closely mimics human COPD.

In general, most genetic alterations including genetic polymorphisms are related to germline inheritance. Therefore, changes in the affected gene expression and/or related gene product function are assumed to occur from the beginning of life, i.e. during development. Severe genetic mutation in some genes that play critical roles during embryogenesis and organogenesis may result in prenatal lethality, which may be related to natural abortion and stillbirth. Moderately severe

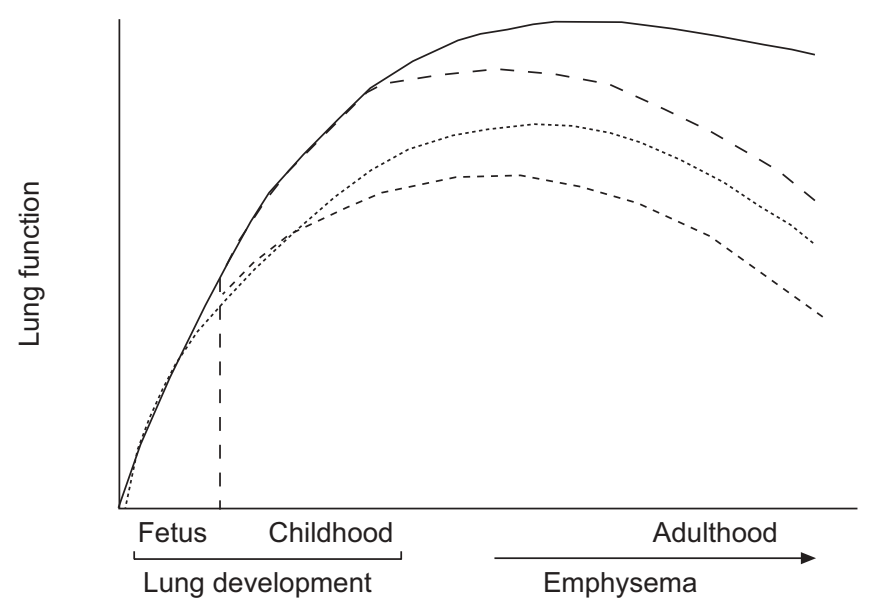

FIGURE 1. A postulated mechanism by which deficiency in some genes may contribute to chronic obstructive pulmonary disease susceptibility, and harmful environmental factors such as smoke exposure exacerbate the pathogenic process. Wild-type lungs growing in room air $(-)$ achieve the greatest functional capacity and thus remain relatively healthy in spite of age related degradation [1]. Smoking exacerbates degradation of lung function, even in healthy individuals (-- ). Genetic alteration, as discussed in this editorial, decreases the potential to develop maximal lung capacity compared with wild type (……..). Smoking exacerbates loss of lung capacity faster, especially in a lung already compromised by genetic mutation (- - -). Also, some steroid-resistant asthmatics fail to develop normal lung functional capacity [10] 
genetic alteration in the genes involved in early development may disturb normal structural formation and function in one or more affected organs/systems, and hence cause clinically diagnosed congenital diseases in children. In addition to the genetic alterations described previously, genetic changes such as DNA polymorphism may only have minor or negligible immediate impact on developing organs/systems, resulting in an apparently normal phenotype in childhood by routine medical examination. However, the affected organ/system may have reduced compensatory capacity and insufficient repair function, leading to a defective response to injury imposed by other factors, including external environmental challenges. As a result, people born with these polymorphisms may be predisposed to certain diseases later in life, so that these diseases appear to have onset during adulthood. Alternatively, genes that are not involved in early development, or that have redundant functions with other genes, may have adverse impacts on certain organs/systems only in adulthood rather than during early development.

In the case of the lung, genetic alteration can affect both normal lung development and function, starting early during the embryonic developmental stage. This may result in both reduction of the eventual maximum lung capacity in terms of gas exchange capacity and lung function, as well as in disturbance of normal lung homeostasis. In particular, upon chronic exposure to harmful environmental factors, such as cigarette smoke or vehicular pollution, the lungs may be predisposed to excessive stress responses, including mechanical and immunological responses in airways and terminal alveolar structures, resulting in activation of pulmonary degenerative cascades that degrade both physiological function and anatomic structure faster than would otherwise occur with normal aging (fig. 1).

\section{STATEMENT OF INTEREST}

None declared.

\section{REFERENCES}

1 Fletcher C, Peto R. The natural history of chronic airflow obstruction. Br Med J 1977; 1: 1645-1648.

2 Sterner-Kock A, Thorey IS, Koli K, et al. Disruption of the gene encoding the latent transforming growth factor-beta binding protein 4 (LTBP-4) causes abnormal lung development, cardiomyopathy, and colorectal cancer. Genes Dev 2002; 16: 2264-2273.

3 Chen H, Sun J, Buckley S, et al. Abnormal mouse lung alveolarization caused by Smad3 deficiency is a developmental antecedent of centrilobular emphysema. Am J Physiol Lung Cell Mol Physiol 2005; 288: L683-L691.

4 Chen H, Zhuang F, Liu YH, et al. TGF-\{beta\} receptor II in epithelia versus mesenchyme plays distinct role in developing lung. Eur Respir J 2008; 32: 285-295.

5 Haitchi HM, Powell RM, Shaw TJ, et al. ADAM33 expression in human lungs and asthmatic airways. Am Rev Respir Dis 2005; 171 : 958-965.

6 Simpson A, Maniatis M, Jury F, et al. Polymorphisms in a disintegrin and metalloproteinase 33 (ADAM33) predict impaired early lung function. Am Rev Respir Crit Care Med 2005; 172: 55-60.

7 van Diemen CC, Postma DS, Vonk JM, et al. A disintegrin and metalloprotease 33 polymorphisms and lung function decline in the general population. Am J Respir Crit Care Med 2005; 172: 329333.

8 Gosman MM, Boezen HM, van Diemen CC, et al. A disintegrin and metalloprotease 33 and chronic obstructive pulmonary disease pathophysiology. Thorax 2007; 62: 242-247.

9 Didon L, Roos AB, Elmberger GP, et al. Lung-specific inactivation of CCAAT/enhancer binding protein $\alpha$ causes a pathological pattern characteristic of COPD. Eur Respir J 2010; 35: 186-197.

10 Covar RA, Spahn JD, Murhy JR, et al. Progression of asthma measured by lung function in the childhood asthma amanagment program. Am J Resp Crit Care Med 2004; 170: 206-207. 